\section{Dr Stählberg comments}

The definition of colic was stated in the introductory lines: the healthy babies had expressed colic of three to 13 hours duration daily, only cases 2 and 6 occasionally having had daily colic of shorter duration than three hours; washout periods have no relevance in this context; any food between treatments might produce carry over effect; professional statisticians were consulted about proper statistical methods.

Based on previous reports ${ }^{1-3}$ we decided that one week on each milk preparation should be sufficient for spontaneous recovery. We knew that only in $50 \%$ of infants would colic resolve by the age of 3 months, particularly children with severe colic who may have extended duration of the problem.

Cases 2 and 9 provided a slight decline in the overall percentage of symptomatic days. Most days colic was present even during the last treatment. A closer look at the information provided in the Tables shows that two children had their longest daily colic during the first treatment, four during the second treatment, two during the third treatment, and two during the last treatment. Three children had their shortest daily colic during the first treatment, three during the second treatment, one during the third treatment, and three during the last treatment. A systematic error by order of treatment is therefore definitely ruled out.

We are sorry for confusing those who were satisfied with the present knowledge about the aetiology of infantile colic. We feel that colic is not a uniform entity nor that milk is the universal cause of the problem. Unfortunately, formulas free from cow's milk are now commonly and uncritically given to infants with colic by health professionals and laymen.

\section{References}

I Jakobsson I, Lindberg T. Cow's milk as a cause of infantile colic in breast-fed infants. Lancet 1978;ii:437-9.

2 Jakobsson I, Lindberg T. Cow`s milk proteins cause infantilc colic in breast-fed infants: a double-blind crossover study. Pediatrics 1983;71:268-71.

3 Evans RW, Fergusson DM, Allardyce RA, Taylor B. Maternal diet and infantile colic in breast-fed infants. Lancet 1981:ii: $134(-2$.

E SAvilahti and M-R Stählberg Turku University Hospital, SF-20520 Turku. Finland

\section{Chromosomal defects and caesarian birth}

Sir.

In their interesting article Dr Young et al provided further evidence that infants with trisomy 18 are often born by caesarian section. ${ }^{1}$ Chromosomal defects were diagnosed after birth in 19 of 1411 infants weighing $501-2000 \mathrm{~g}$ liveborn to Merseyside residents in 1979-1981. Eleven $(58 \%)$ of the 19 were delivered by caesarian section-five elective and six during labour-compared with $27 \%$ of the other infants $(\mathrm{p}<0 \cdot 01)$.
The increased risk of caesarian birth was significant after allowing for birth weight, gestational age, and sex $(p<0 \cdot(05)$. The risk, however, applied not only to infants with trisomy 18 (five of aight) but also to infants with other chromosomal defects (six of 11 infants). These comprised all infants with monosomy $4 p(n=1)$, trisomy $4 p(n=1){ }^{2}$ ring $13(n=1)$, and trisomy $13(n=1)$, and two of seven with trisomy 21 .

The risk was related to severe growth retardation: the birth weights of nine of the 11 born by caesarian section were below the 5 th centile for gestational age compared with two of the eight born vaginally $(\mathrm{p}<0) \cdot(05)$; trisomy 21 was not associated with severe growth retardation as often as the other defects. Infants with chromosomal defects had lower 5- and 10-minute Apgar scores than other infants $(p=0 \cdot(2)$ and $p=0 \cdot() 11$, respectively); associated cardiac defects may increase the risk of fetal distress and obstetric intervention.

The authors point out the potential value of a safe means of rapid fetal karyotyping in late pregnancy, when growth retardation or hydramnios raise suspicion of a severe congenital defect. Our low birthweight sample, however, contained 560 growth retarded livebirths (birthweight $<10$ th centile for gestational age) of which only 13 had diagnosed chromosomal defects. Would ultrasonography for cardiac defects help to narrow the field?

\section{References}

1 Young D. Cook JP, Metha L. Arch Dis Child 1986;61:1035-6.

2 Borgaonkar DS. Chromosomal variation in man. A catalog of chromosomal variants and anomalies. New York: Alan R Liss, 1984.

G Powell. P O D Pharoah, and R W I Cooke Departments of Community Health and Child Health, University of Liverpool, Liverpool $L 693 B X$

\section{Phosphatidylglycerol in tracheal aspirates for diagnosing hyaline membrane disease}

Sir,

In a recent paper,' we showed that the simple and rapid determination of phosphatidylglycerol (PG) in tracheal aspirates, for the diagnosis of hyaline membrane disease. was more sensitive $(97 \%)$ than the lecithin:sphingomyelin (L:S) ratio $(88 \%)$, with a similar specificity $(76 \%)$. It seems to us, however, that the discrepancy between biochemical tests and clinical diagnosis was observed particularly in the lowest gestational age newborns.

Accordingly, we studied PG and the L:S ratio in 149 newborns with respiratory disease (term, 26 to 42 weeks) to determine whether there was a correlation between the results and the gestational age.

For the L:S ratio, the results did not depend on the gestational age. The sensibility and specificity of this test were $91 \%$ and $74 \%$, respectively. With the determination of PG, however, the results depended on the gestational age. For newborns with a gestational age of 31 weeks or 